\title{
Successful anesthetic management of a living-donor liver transplant for a patient with severe methylmalonic acidemia: A case report.
}

\author{
Akiko Hirotsu, M.D., Eriko Kusudo, M.D., Natsumi Mori, M.D., \\ Yoshimitsu Miyai, M.D., Kengo Suzuki, M.D., Ph.D, Shuji Kawamoto, M.D., Ph.D. \\ Department of Anesthesia, Kyoto University Hospital
}

Background:

Few reports are available on anesthetic management of liver transplantation (LT) for methylmalonic acidemia (MMAemia) [1], although LT has been performed as a supportive therapy for severe MMAemia to improve the prognosis. Here, we show the successful perioperative management of a MMAemia patient receiving LDLT.

Case presentation:

22-month-old girl $(70 \mathrm{~cm}, 9.9 \mathrm{~kg})$ with severe MMAemia was admitted to our hospital to have a living donor LT from her mother.

[Preoperative condition and management]

\begin{tabular}{|c|c|}
\hline $\begin{array}{l}\text { positive symptoms } \\
\text { - recurrent vomiting } \\
\text { - seizures } \\
\text { - mental \& developing } \\
\text { retardation } \\
\text { negative symptoms } \\
\text { - renal dysfunction }\end{array}$ & $\begin{array}{l}\text { - } \quad \text { protein restricted diet } \\
\text { continuous infusion of } \\
\text { glucose, vitamin } B_{12} \\
\text { and L-carnitine (DIV) } \\
\text { - } \text { medication } \\
\text { (anticonvulsant } \\
\text { agents) }\end{array}$ \\
\hline
\end{tabular}

【Preoperative Laboratory data】

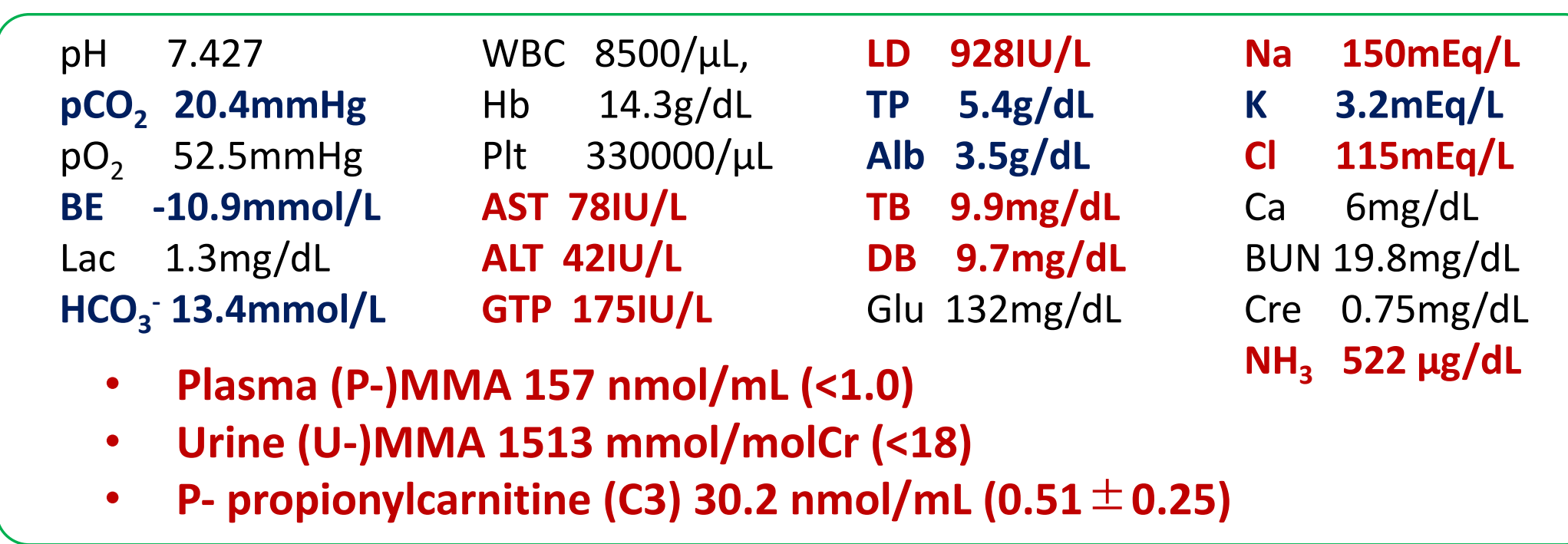

【Anesthetic summery of LDLT】

- Preoperative fasting: $6 \mathrm{hrs}$ before induction.

- Continuous hemodiafiltration (CHDF) was prepared in advance.

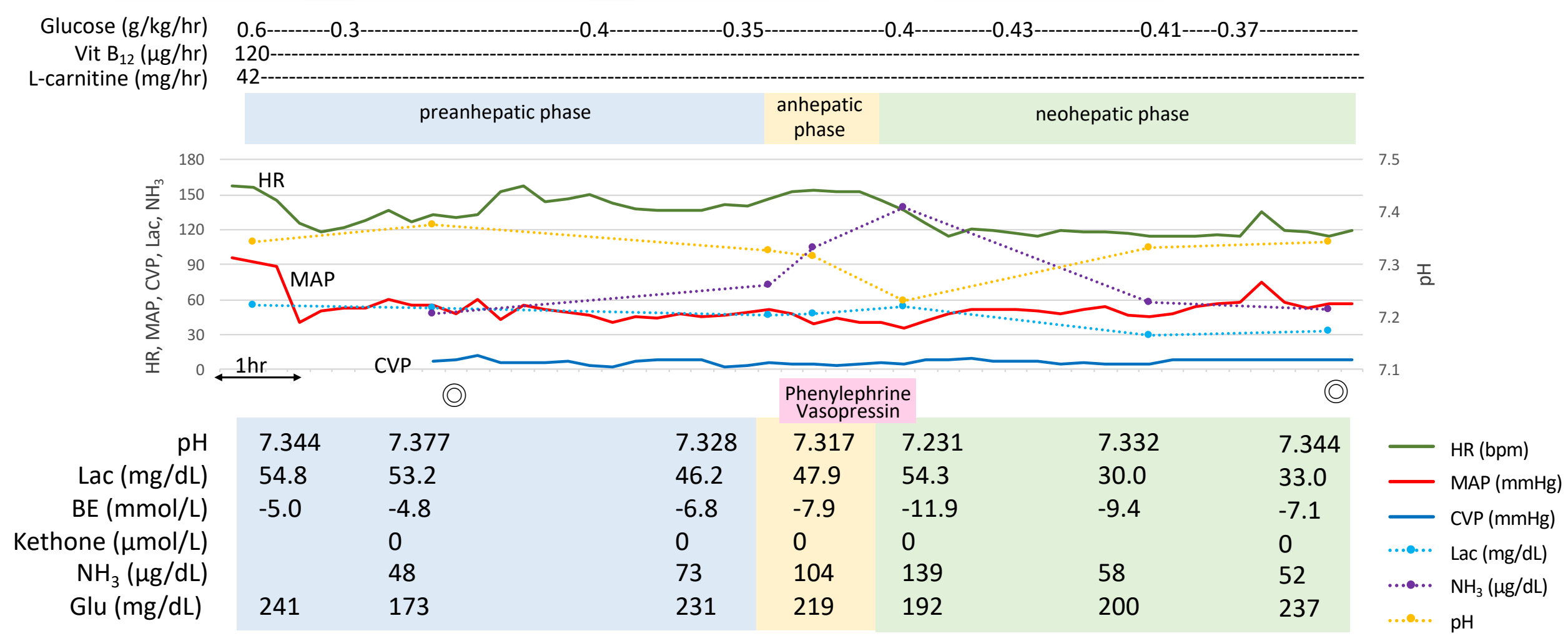

【Postoperative course】

- Plasma MMA concentration decreased at 7 POD (table 1).

- Extubated at 3 POD and discharged at 80 POD (additional management for acute rejection was needed before discharge.)

Discussion:

1. The anesthetic management of LT for MMAemia is challenging because preoperative fasting and surgical stress can worsen metabolic acidosis in MMAemia patients, which can be further worsened during the anhepatic phase in liver transplantation.

2. Metabolic decompensation during LT is related to a high risk for mortality and severe postoperative metabolic complication [2]. Therefore, anesthetic management is important and if metabolic acidosis worsened during LT, further treatment should be started without delay.

3. Long-term prognosis of our patient cannot be clearly predicted, because the MCM activity in the transplanted graft from the heterozygous donor should be less than normal.
- Anesthetic drugs: fentanyl, remifentanil, midazolam, sevoflurane, and rocuronium

- Total fluid infusion: $1175 \mathrm{~mL}$

- Blood loss: low amount

- Urine output: $135 \mathrm{~mL}$

- Metabolic acidosis improved and $\mathrm{NH}_{3}$ level decreased rapidly after reperfusion, and LDLT was successfully performed without CHDF.

\begin{tabular}{|cccc|}
\hline & $\begin{array}{c}\text { U-MMA } \\
(\mathrm{mmol} / \mathrm{mol} \mathrm{Cr})\end{array}$ & $\begin{array}{c}\mathrm{P}-\mathrm{MMA} \\
(\mathrm{nmol} / \mathrm{mL})\end{array}$ & $\begin{array}{c}\mathrm{P}-\mathrm{C} 3 \\
(\mathrm{nmol} / \mathrm{mL})\end{array}$ \\
\hline pre ope & 1513 & 157 & 30.2 \\
\hline 7 POD & 165 & 18.7 & 8 \\
\hline
\end{tabular}

Table 1. Transition of plasma and urine MMA, and plasma C3 concentration

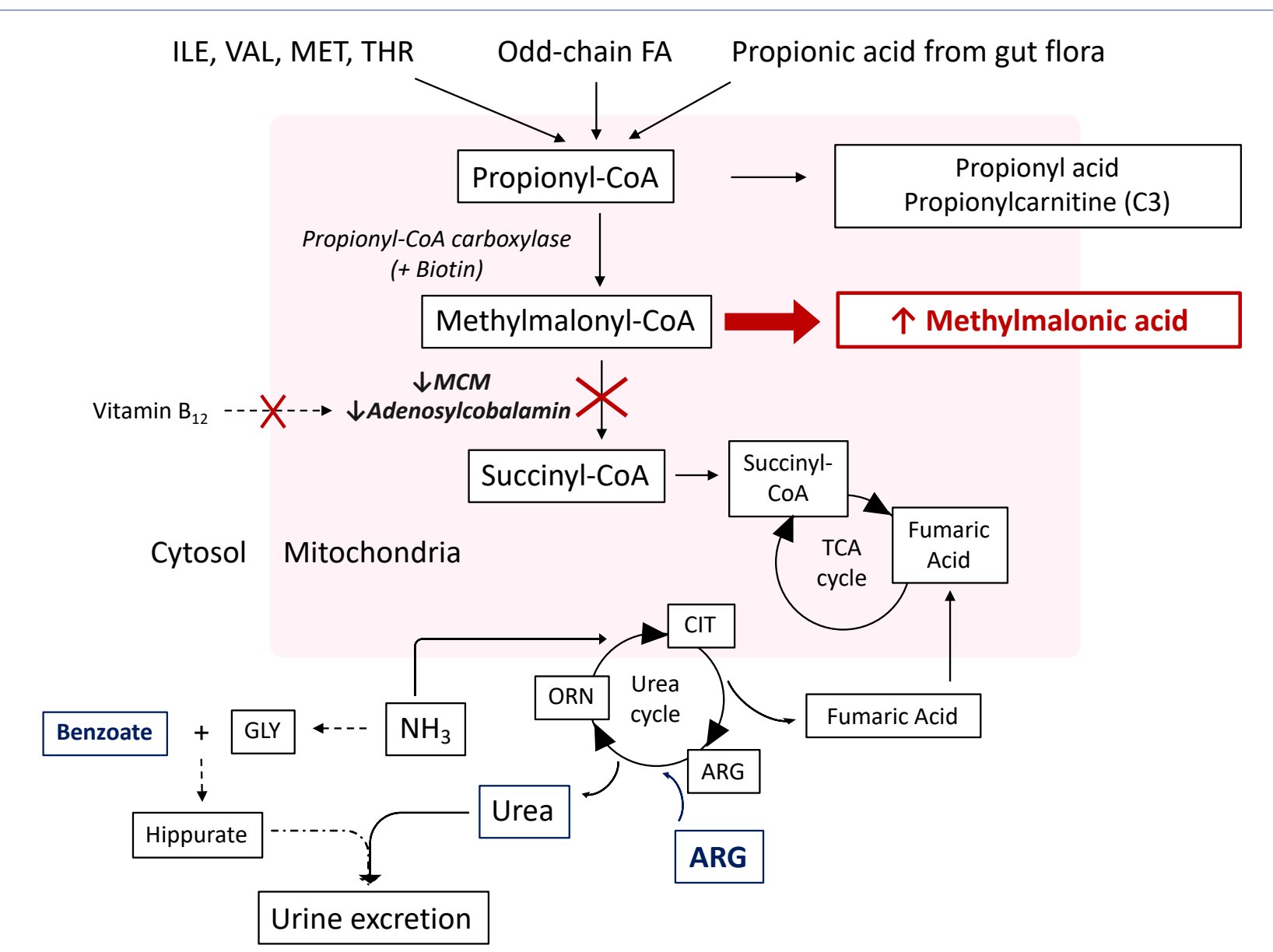

Figure: Pathophysiology of MMAemia and excess nitrogen excretion

(ILE: Isoleucine, VAL: Valine, MET: Methionine, THR: Threonine, FA: Fatty acid, MCM: MethylmalonylCOA mutase, TCA: Tricarboxylic Acid, CIT: Citrulline, ORN: Ornithine, ARG: Arginine, GLY: Glycine) 